\title{
Global accessibility of therapeutics for diabetes mellitus
}

\section{Michael Fralick, Alicia J. Jenkins, Kamlesh Khunti, Jean Claude Mbanya, Viswanathan Mohan (D) and Maria Inês Schmidt}

Diabetes mellitus is a global health issue, yet huge regional disparities exist in its care, including in access to basic necessities such as insulin. In this Viewpoint, six experts from different regions discuss differences in access to insulin and other diabetes mellitus therapies as well as the key barriers in diabetes mellitus care accessibility and potential solutions.

\section{Q How accessible is insulin in your region?}

Michael Fralick (Canada and the USA). Insulin was first successfully administered on January 23, 1922, at the Toronto General Hospital in Ontario, Canada. Mere months thereafter, the patent for insulin was signed over to the University of Toronto for $\$ 1$. The reason, as stipulated by Dr Frederick Banting, was simple: "Insulin does not belong to me, it belongs to the world." Yet, despite this altruistic principle present at the inception of its clinical use, the painful irony is that presently (and historically), the cost of insulin is one of the single greatest barriers to its access in the USA and many other parts of the world ${ }^{1}$.

In the USA, the cost of, and therefore access to, insulin depends primarily on whether a person has health insurance. The 27 million US residents living without medical insurance pay for medications out-of-pocket, and insulin is no exception. Even among US residents who have health insurance through government programmes such as Medicare (for example, for people aged 65 years and older) or Medicaid (for people with low income), there remain substantial out-of-pocket costs incurred. Multiple studies have demonstrated that the high costs of insulin and its associated supplies have led to the underutilization of insulin in the USA, including the practice of insulin dose rationing, in which people living with diabetes mellitus underdose themselves in an effort to make their insulin supply last longer ${ }^{2,3}$. It is estimated that one in four US residents living with diabetes mellitus engages in this undesirable practice because of the high cost of insulin ${ }^{2,3}$. Many Americans living close to Canada drive across the border to purchase their insulin because the cost of insulin in Canada, remarkably, is approximately one-tenth of what it costs in the USA ${ }^{1}$.

\section{Jean Claude Mbanya (sub-Saharan}

Africa). Eighty percent of people living with diabetes mellitus live in developing countries, yet only $1 \%$ of global diabetes-related expenditure occurs in these countries. The availability of insulin has shifted the focus of diabetes mellitus treatment from trying to keep patients alive, to improving the quality of life of those with the condition. However, a 2019 study in 13 sub-Saharan African countries found that the mean availability of human insulin was $55-80 \%$ in facilities that should have had insulin available on the day of study. Moreover, poor availability of insulin was found in both the public and private health-care sectors as well as at different levels of the health system: for example, insulin was more available at hospitals than at primary health-care centres ${ }^{4}$. Only 2 of the 13 African countries met the WHO's global action plan on non-communicable diseases (NCDs) target of $80 \%$ availability of essential medicines, which includes insulin. People with diabetes mellitus in sub-Saharan Africa continue to die prematurely because of the lack of access to insulin. In rural and urban Mozambique, a child diagnosed with type 1 diabetes mellitus (T1DM) had a life expectancy of $<1$ year and 4 years, respectively. Therefore, having a child with T1DM in a family is like a death sentence if the family cannot afford the cost of insulin. This has led many patients to ration their daily doses of insulin to last until the family can afford to purchase the next vial.

Viswanathan Mohan (India). The prevalence of diabetes mellitus is growing rapidly the world over and especially in developing countries, where about $80 \%$ of all people with diabetes mellitus now live. Indeed, India and China alone account for about $40 \%$ of the global diabetes mellitus burden. Currently, India is home to 74 million people with diabetes mellitus ${ }^{5}$. During the 100th year since the discovery of insulin, it is sad that, in many parts of the globe, insulin is not accessible, available or affordable. In India, luckily, insulin is available and accessible in many areas, except perhaps in some of the mountainous regions and villages of a few remote states of the country. However, the fact remains that insulin is still not affordable for many. Paradoxically, it is the people belonging to the middle-income group, who comprise about $70-80 \%$ of the population of India, who are affected most. For this group of people, the medical expenses are 'out-of-pocket'. People who live below the poverty line are usually provided free insulin by the government, while the affluent can afford to buy even the most expensive insulin. Hence, there is a huge economic divide with respect to accessibility to insulin in India.

Alicia J. Jenkins (Oceania). Diabetes mellitus affects people of all ages, genders, ethnicities and religions, and of any educational and socioeconomic status; however, the prognosis varies greatly between and within countries. In Oceania, the incidence and prevalence of diabetes mellitus continue to rise, affecting about $10 \%$ of people, with rates as high as $22 \%$ in Kiribati ${ }^{5}$. The personal and socioeconomic costs are enormous.

In Australia, a person can live with T1DM for over 70 years, have subsidized access to hybrid-closed-loop insulin pumps, a range of modern human insulins and glucagon, continuous glucose monitors, home glucose and ketone monitoring, and medications for risk factor control and for the primary and secondary prevention 
of diabetes mellitus complications.

Vascular surgery, dialysis, sight-preserving intraocular injections and organ transplants are available and often subsidized. An Australian person with T1DM can usually access a skilled multidisciplinary team of health-care professionals in-person and by telehealth, though less readily if they live in rural or remote areas. This same condition occurring in a child elsewhere in Oceania might lead to an early death due to misdiagnosed diabetic ketoacidosis. It is estimated that, for every two people living with T1DM globally today, one has died due to lack of access to even basic care. An estimated one in two people with type 2 diabetes mellitus (T2DM) in Oceania do not know they have the condition ${ }^{5}$; however, not knowing does not protect them from developing many acute and chronic complications. In some regions of Oceania, even knowing that one has diabetes mellitus and needs education, tests and medicines, does not mean that one can access them. Affordability is part of accessibility. As Charles Dickens wrote (in A Tale of Two Cities): "It was the best of times, it was the worst of times". Such is the case for diabetes mellitus care in Oceania.
Q How accessible are other drugs for diabetes mellitus in your region?

\section{Maria Inês Schmidt (South America).}

Based on the most recent survey carried out by the Pan American Health Organization (PAHO) in 2020 in 35 countries in the Americas, medicines for diabetes mellitus care are considered as available (that is, available in $>50 \%$ of the pharmacies within the public sector); specifically, insulin is available in $87 \%$ of the countries, metformin in $94 \%$ of the countries, sulfonylureas in $91 \%$ of the countries, and other essential medicines for diabetes mellitus management are usually available in $>80 \%$ of the countries. Undoubtedly, this is insufficient, and much more is needed. The challenge is enormous, particularly considering the rapid epidemiological transition from predominantly infectious and parasitic diseases to chronic conditions. Moreover, countries must now cope with the huge economic effects and impact on health of the COVID-19 pandemic.

To address the topic more specifically, I will consider Brazil, where I live and work. Brazil is a large middle-income country, where 210 million people live in a territory

\section{The contributors}

\section{Michael Fralick}

Michael Fralick is a board-certified General Internist in Canada and the USA. Currently, he is a clinician scientist at Sinai Health in Toronto and Assistant Professor at the University of Toronto's Faculty of Medicine. He also works clinically at the Sault Area Hospital in Sault Ste. Marie, Ontario, Canada.

\section{Alicia J. Jenkins}

Alicia Jenkins is an adult endocrinologist and a clinician-researcher at The University of Sydney, Australia. Her clinical and research interests include the prediction and prevention of diabetes complications and technology use in diabetes mellitus care. She is also a Board Member of Insulin For Life (www.insulinforlife.org), a diabetes aid NGO.

\section{Kamlesh Khunti}

Kamlesh Khunti is a general practitioner and Professor of Primary Care Diabetes and Vascular Medicine at the University of Leicester, UK. He has been a recipient of numerous international awards, published over 1,000 articles in peer-reviewed journals and was named one of the top researchers in type 2 diabetes mellitus by Expertscape.

\section{Jean Claude Mbanya}

Jean Claude Mbanya, MD, PhD, FRCP (London), is a Professor of Medicine and Endocrinology and Postgraduate Dean, Doctoral School of Life Sciences, Health and Environment at the University of Yaoundé I, Yaoundé, Cameroon.

\section{Viswanathan Mohan}

Viswanathan Mohan, MD, PhD, DSc, FRSE, is Chairman of Dr Mohan's Diabetes Specialities Centre and Director of Madras Diabetes Research Foundation at Chennai, India. He is the recipient of many international awards, including the Dr Harold Rifkin Award from the American Diabetes Association. Dr Mohan has over 1,400 publications with over 161,000 citations and an h-index of 141 .

\section{Maria Inês Schmidt}

Maria Inês Schmidt is a Professor at the School of Medicine, Universidade Federal do Rio Grande do Sul, Brazil. She was part of various diabetes mellitus panels for WHO and is a member of the Lancet Commission for Diabetes and of the Brazilian Academy of Science. She trained in Paediatric Endocrinology at the Johns Hopkins Hospital, Baltimore, MD, USA, and in Epidemiology at the University of North Carolina, Chapel Hill, NC, USA.

of 8.516 million $\mathrm{km}^{2}$. Public policies aimed to confront NCDs started to be implemented in the 1980s, the most successful being tobacco control; smoking rates have fallen from close to $40 \%$ to currently $~ 10 \%$ of the adult population ${ }^{6}$. A more structured national plan to confront NCDs was implemented in 2011 but did not include a specific target to reduce diabetes mellitus prevalence. Other relevant interventions are a dietary guideline aiming to decrease the consumption of ultra-processed foods and beverages (2014) and a physical activity guideline (2021).

Although progress in reducing age-standardized premature deaths due to NCDs has been observed in Brazil, the rate of fall has recently plateaued and trends in cardiometabolic risk factors are generally stable or worsening, and the burden of diabetes mellitus is on the rise. An estimated 9.1 million persons had known diabetes mellitus in 2013 in Brazil, representing approximately $70 \%$ of the total cases in Brazil $^{7}$. Among known cases of diabetes mellitus, only an estimated $46 \%$ had $\mathrm{HbA}_{1 \mathrm{c}}$ levels of $<7 \%$. Moreover, $34 \%$ had $\mathrm{HbA}_{1 \mathrm{c}}$ levels above $8 \%$. These figures, and those regarding the control of other risk factors, indicate that much more is needed in diabetes mellitus management in Brazil ${ }^{8}$. In addition to privately financed care, Brazil has a universal health system (Sistema Unico de Saúde), with a public primary care network available to all and covering $63 \%$ of the population in 2021. Essential medicines and tools for the care of diabetes mellitus have been progressively made available free of charge, including insulin analogues and pens in 2021 for specific groups living with T1DM. Additionally, a sodium-glucose transport protein 2 (SGLT2) inhibitor is now approved for those with T2DM at the highest risk. A digital health system has also been partially implemented.

V.M. The older antidiabetic drugs, like metformin and sulfonylureas, are inexpensive and are available in most parts of India 9 . However, the newer agents (such as the dipeptidyl peptidase 4 (DPP4) inhibitors, SGLT2 inhibitors and glucagon-like peptide 1 (GLP1) receptor agonists) are more expensive and hence are unaffordable by the majority of people in India. Luckily, this problem has been offset to some extent by the introduction of generic drugs by innovative Indian pharmaceutical companies. Teneligliptin, a low-cost DPP4 inhibitor from Japan, was introduced in 2015 as a generic drug followed later by remogliflozin, a generic SGLT2 inhibitor. 
In 2019, vildagliptin (another DPP4 inhibitor) went off patent and by 2020 several Indian companies introduced this molecule in generic form, making it affordable. Finally, a generic version of the SGLT2 inhibitor dapagliflozin was also introduced in 2021. Thus, oral hypoglycaemic agents in general are available, accessible and affordable in our region.

J.C.M. In 2015, the WHO found that, in Africa, only $51 \%$ of the countries had metformin routinely available. Efficient procurement of medicines is critical to ensure that patients with diabetes mellitus within public health-care systems are able to obtain an uninterrupted supply of their medicines. There are also concerns with available resources and co-payments within public health-care systems in Africa, leading to continued endorsement and listing of appropriate sulfonylureas and metformin rather than funding newer oral medicines such as DPP4 and SGLT2 inhibitors. Furthermore, there are concerns with clinical inertia, with physicians prescribing insulins rather than again funding newer and more expensive oral antidiabetic medicines.

Kamlesh Khunti (Europe). There are approximately 57 countries and territories in the European Region, and the International Diabetes Federation Atlas in 2021 estimated that there were 61 million people with diabetes mellitus across Europe ${ }^{5}$. The prevalence of diabetes mellitus in adults is approximately $8.9 \%$ in Europe, with the costs amounting to 161 billion Euros. Most countries in Europe have some form of guidelines on diabetes mellitus, the majority using a combination of National and International guidelines, with a substantial degree of consensus for specific targets in European guidelines.

Prescription medication in European countries is very well regulated and, overall, Europe has fair access to medication and health-care services. However, once approved, patients' access to medications is not always guaranteed due to a number of factors. For example, whether the pharmaceutical companies market the medicine in that specific country, whether the national health system agrees to reimburse the medication costs, the affordability of the medication depending on the level of co-payment required, and whether the local pharmacy will make the medicines available. European countries all vary in how they manage these key factors of accessibility for their patients, with each country having different approaches to medication reimbursement. In view of these policy variations, access to medications is not equal across European populations. Furthermore, the prevalence of diabetes mellitus, and therefore the health-care costs for diabetes mellitus, in different European countries varies considerably.

One study examined the uptake of new medicines in 11 European countries over a 10 -year period from 2006 to 2016, during which 14 new active medications were introduced ${ }^{10}$. Overall, the median time for continuous use of the medications for different countries was 13 months, but this varied substantially, with the fastest uptake in Germany and the UK (with the median time of 3 months) and the longest in Croatia (with the median time of 30 months). This study also showed that, overall, there was a decrease in the market share volume for old glucose-lowering medications, with the share of new medications increasing over the 10 -year period. There were substantial variations, however, with the uptake of new glucose-lowering medications being $9.7 \%$ for Sweden and $26.3 \%$ for Spain.

European countries also differ hugely in the extent of insulin use. For example, Slovenia, Germany and Sweden have a market share of at least $30 \%$ of insulin use compared with lower market shares in countries such as France, Croatia, Italy and the UK. Access to more innovative therapies, such as insulin pump therapy, also varies widely. For example, in 2013, the UK reported that $97 \%$ of hospitals provided insulin pump services, although only $7 \%$ of the estimated population of people with T1DM in the UK was using insulin pump therapy. This contrasts with countries such as Germany and Norway, where over 15\% of their population with T1DM use pump therapy, and the USA, where $40 \%$ of people with T1DM use pump therapy. Data from Europe and USA also show that basal insulin is initiated late (mean $\mathrm{HbA}_{1 \mathrm{c}}$ level of 9.0\%) in people with $\mathrm{T} 2 \mathrm{DM}$, with the majority of patients not reaching an $\mathrm{HbA}_{1 \mathrm{c}}$ level of $\leq 7 \%$ in the first 3 months of basal insulin therapy or after 2 years of basal insulin therapy. Therapeutic inertia has been identified as the key barrier in diabetes mellitus care, with delayed glycaemic control and failure to achieve T2DM targets.

M.F. Canada, unlike the USA, provides universal access to health care; however, access to prescription medications is not universal in Canada. Among Canadian adults who are currently working, approximately two-thirds have private drug coverage that is either employer based or paid for out-of-pocket. For specific populations, Canadian governments do provide support for prescription medications through government assistance programmes: most adults over 65 years of age have essentially no out-of-pocket costs for prescription medications apart from a small co-pay amount. For example, in Ontario (Canada's most populated province), adults aged 65 years and older or under 25 years of age pay a fee of approximately CDN $\$ 5$ per month, which covers the cost of all of their prescription medications for the month. For those aged 25-65 years, people generally pay out-of-pocket or through private insurance, which is most often employer based. Additionally, for those of very low socioeconomic status or those living with disabilities, there are essentially no out-of-pocket costs for prescription medications on the Ontario formulary. Unsurprisingly, access to insulin in Ontario - and across Canada - is much better than it is in the USA.

In the USA, accessibility of diabetes mellitus medications other than insulin is also directly tied to cost. A 1-month supply of generic diabetes mellitus medications (like metformin or glimepiride) costs about US $\$ 3$, and is sometimes provided free of charge at pharmacies to attract new clients. Access to these generic medications has few financial barriers. By contrast, for brand name medications that lack a generic alternative, the costs can be prohibitive. For example, for US residents without insurance, the approximate cost of a 1-month supply of sitagliptin is US $\$ 500$, empagliflozin is US $\$ 600$ per month and liraglutide is US $\$ 1,200$ per month. Although the cost is lower for US residents with health insurance, there are other barriers to accessing certain (often newer) diabetes mellitus medications. For instance, in order to prescribe a new medication to a person, a US health-care provider often has to complete additional, frequently complex, paperwork for the insurance company such as prior authorization forms.

\section{Q What is the main problem regarding diabetes mellitus management in your region?}

A.J.J. The major problem regarding diabetes mellitus management in Oceania is inequitable access to medicines and other care, including devices, diagnostics, therapeutic procedures and skilled care teams. Diabetes mellitus education and a healthy lifestyle are key for all people with diabetes mellitus as they usually spend 
only a few hours per year $(<0.02 \%$ of time $)$ with health-care professionals and must manage multiple aspects of their health on a daily basis. Even though insulin has been on the WHO Essential Medicines Lists for decades, insulin availability, even for people with T1DM, is not always assured in some countries. Even in affluent countries, the availability of insulin and other drugs does not always equate to accessibility, as some medicines and devices (such as health-improving insulin pumps and continuous glucose monitoring) are not fully subsidized. In some countries, drug availability and expert care teams are concentrated in major cities, contributing to worse outcomes for people living in rural or remote regions. Obesity and T2DM are more common in lower socioeconomic groups. Many people with T2DM require ten or more tablets a day, for glucose, blood pressure and lipid control, and medicines for other health conditions; thus, even with subsidies, people cannot always afford their recommended and desired treatments.

The costs of even basic diabetes mellitus care today remain well beyond the reach of many families ${ }^{11,12}$, communities, health-care systems and countries in Oceania and other parts of the world. As Elliot Joslin said in 1928, "the effort of diabetes care is great, but the reward is also great, for the prize is life itself".

J.C.M. Beyond access to diabetes mellitus medication, a variety of health system factors further hamper access to care in sub-Saharan Africa. These include diabetes mellitus care being provided at hospitals versus in primary health care, lack of knowledge and expertise of health professionals with regard to insulin therapy, and the availability and affordability of diabetes mellitus-related supplies. These health system factors can also affect the overall cost of diabetes mellitus management. For example, individuals might need to pay for travel from their home to a facility where insulin is present, or might only be able to access this medicine in the private sector at higher prices.

There is also inadequate availability of simple equipment for diagnosis and monitoring, a lack of sufficiently knowledgeable health-care providers, insufficient availability of treatments, a dearth of locally appropriate guidelines and few disease registries. These inadequacies result in a substantial drop off of patients along the diabetes mellitus care cascade, with many patients going undiagnosed and with those who are diagnosed not receiving the advice and drugs they need ${ }^{13}$.
M.F. The primary reason why drugs cost more in the USA is that there are essentially no regulations moderating how much a medication can be sold for, or how much the price can increase each year ${ }^{14}$. By comparison, in Canada, the costs of medications (brand name and otherwise) are lower than in the USA, owing to Canada's stricter drug pricing regulations. Canadian residents who pay out-of-pocket can expect to pay about one-fifth of what their US counterparts pay for most brand name medications. There are also less administrative hurdles in Canada for clinicians to prescribe newer, brand name diabetes mellitus medications.

Despite the lower costs and fewer administrative obstacles in Canada, one of the main problems regarding diabetes mellitus management in both Canada and the USA is the underutilization of newer diabetes mellitus medications such as SGLT2 inhibitors and GLP1 receptor agonists. Both classes of medications have been shown to improve cardiovascular and renal outcomes, and SGLT2 inhibitors have also been shown to improve heart failure outcomes ${ }^{15}$. The reasons for the slow uptake of these new medications are unclear but are probably related to prescribing inertia and a lack of knowledge or familiarity. For people living with T2DM, there are more effective treatments available now than ever before. However, for their clinicians, the number of available treatment options might be overwhelming. Knowledge translation tools are an important bridge in the knowledge gap and might disrupt prescribing inertia and increase the utilization of SGLT2 inhibitors and GLP1 receptor agonists, which are known to improve patient outcomes.

Another important problem regarding diabetes mellitus management in both Canada and the USA is differential access to care based on geography, ethnicity and socioeconomic status. For example, people living in rural areas or First Nations individuals have worse outcomes compared with people living in urban areas or white individuals.

V.M. There are several challenges of diabetes mellitus management in India. These challenges include the large size of the country and the facts that almost $50 \%$ of people with diabetes mellitus remain undiagnosed and over $70 \%$ of medical care is private, which translates into lack of affordability of treatment by many. Finally, there is an abundance of unqualified practitioners, who lure people away from insulin to unproven therapies by spreading fake news about the safety of modern scientific medicines. Quite often, people have to travel large distances to reach a specialized diabetes mellitus clinic. This means that an annual check-up of the eyes, kidneys, heart and feet, which is considered part of routine diabetes mellitus care nowadays, becomes difficult. Not surprisingly, there is a huge burden of diabetic complications in India ${ }^{16}$. A unique challenge in India is that allopathic (modern scientific) medicines, including antidiabetic drugs, are generally perceived to be harmful if taken on a long-term basis. There are several alternative systems of medicines in India (for example, Ayurveda, Siddha, Unani and homeopathy), which claim to 'cure' diabetes mellitus. These systems portray allopathic drugs as agents, which only 'mask' the symptoms of the disease in addition to being toxic, as they are referred to as 'chemicals'. This deep-rooted mistrust of allopathic medicines leads to people stopping their medications once they become asymptomatic.

K.K. Despite good access to medications, the achievement of guideline-derived targets for $\mathrm{HbA}_{1 \mathrm{c}}$ are low in Europe, although slightly higher compared with a global target achievement (50\% versus $42.8 \%)^{17}$. The DISCOVER study, involving nearly 16,000 patients with T2DM, showed that second-line therapy with a DPP4 inhibitor on the background of metformin therapy was the most commonly prescribed therapy in Europe ${ }^{18}$. However, the mean $\mathrm{HbA}_{1 \mathrm{c}}$ in Europe on initiation of second therapy was $8.1 \%$ compared with $8.3 \%$ for the overall global population, demonstrating therapeutic inertia. Furthermore, despite high-quality cardiovascular outcome trials and recommendations by international guidelines, there are still large variations in uptake of evidence-based cardioprotective glucose-lowering medications across Europe. The uptake of medications is dependent on restrictions that are guided by country-specific guidelines, with Germany being the fastest at launching new agents. In some European countries, prescribing is also restricted and can only be done by specialists while, in other countries such as the UK and Spain, primary care practitioners can prescribe all therapies. Indeed, certain therapies are still not available in all countries such as the unavailability of SGLT2 inhibitors in France. There are also restrictions in terms of which patients have access to medications, for example, GLP1 receptor 
agonists in the UK can only be prescribed to people with obesity, while there are no such restrictions in some other European countries.

Q What action can be taken now to improve access to insulin and other diabetes mellitus drugs for patients?

J.C.M. All solutions for improving access to insulin need to have people with diabetes mellitus playing a key role and should include innovations that decrease global inequalities. Different players will need to be involved, including the WHO, diabetes associations, academia and the private sector. International support is needed to advance WHO prequalification (a process to determine the capacity of a manufacturer to produce insulin and other medications in accordance with international standards), strengthen national regulatory agencies and improve processes surrounding the selection, pricing, reimbursement, procurement and supply of insulin. The WHO or other organizations within the UN can also develop a pooled procurement mechanism for insulin and diabetes mellitus management-related supplies. In addition, global clinical and scientific diabetes mellitus societies have a role in the development of context-appropriate and conflict-of-interest-free guidelines and training. National governments can develop comprehensive policies on the selection and pricing of products for diabetes mellitus management, must ensure insulin and other diabetes mellitus supplies are included in universal health coverage packages, and should remove or regulate mark-ups within the supply chain. Overall, health systems and the way in which diabetes mellitus care is delivered needs to be strengthened, ensuring that it is centred on the needs of people with diabetes mellitus.

The private sector has a clear mandate, which is included in the UN's Sustainable Development Goals, and can play a role in addressing the challenge of access to insulin. These initiatives should focus on creating true partnerships with other players and addressing the issue of the price of insulin in a sustainable way. As aforementioned, one area that needs to be further strengthened at a national and global level is civil society mobilization. Although global and national diabetes mellitus organizations exist and provide training, guidelines and education, so far, they have failed to materialize into a truly global movement and ensure that the voice of people with diabetes mellitus is heard ${ }^{19}$.
M.I.S. To respond to this challenge of diabetes mellitus management globally, PAHO launched the Global Diabetes Compact for the Americas in the wake of the WHO launch of the Global Diabetes Compact. The initiatives call on nations to improve diabetes mellitus prevention and care, asking countries to set their targets and plans for diabetes mellitus care. An accompanying module, referred to as HEARTS-D, is directed to primary health care on diagnosis and management of T2DM, as part of the HEARTS

Technical Package. HEARTS-D is aligned with the WHO Package of Essential Noncommunicable Disease Interventions in Primary Health Care (WHO-PEN).

A few minimal standards are mandatory. Regarding T1DM, it is essential to guarantee universal access to basic care, including human insulin, nutritional and educational support, self-monitoring, $\mathrm{HbA}_{1 \mathrm{c}}$ determinations, and clear mechanisms for referral to specialized treatment when needed. Regarding T2DM, it is necessary to provide access to basic care, including metformin, sulfonylureas and human insulin, risk factor control, including antihypertensive drugs and statins, and nutritional support.

According to local priorities and resources, data-informed steps can be taken progressively as addressed by a comprehensive document prepared by the Lancet Commission on Diabetes ${ }^{1}$. For example, for T1DM, team management, supply of insulin analogues and pens to specific groups, and more frequent glucose monitoring could be implemented. For T2DM, the addition of antidiabetic drugs (such as SGLT2 inhibitors and GLP1 receptor agonists), support for major weight loss for newly diagnosed individuals, and programmes to enhance physical conditioning are good options. The detection of unknown diabetes mellitus and lesser states of hyperglycaemia could also be undertaken if adequate treatment can be provided to those diagnosed. Health system improvements are necessary, including the implementation of a chronic care model, digital tools to monitor and evaluate care, and telehealth. Country-level monitoring of the rate of undetected diabetes mellitus and of the incidence of diabetes mellitus and its complications should be obtained from survey data, and/or progressively derived from digital health and diabetes mellitus registries that are linked with other health system records.

The task ahead is huge and involves multiple actors at the national and global levels. National investments in universal primary care health systems are usually not that large and their benefits can be extended to other aspects of health beyond diabetes mellitus. However, investments in new and extremely expensive drugs can be huge, are frequently unaffordable and must therefore be a focus of global action involving all stakeholders.

So, what could the next steps be? To effectively decrease inequities in care, I strongly recommend strengthening health systems. A minimal digital model of care for the management of those with T1DM and T2DM must be established. This system should enable the monitoring of selected outcome indicators, among them the percentage of people with $\mathrm{HbAlc}$ levels of $>8 \%$, as well as the incidence of hyperglycaemic crises and chronic complications. Programmes to support lifestyle changes, including those aiming at major weight loss during the initial phases of T2DM to minimize the need for multiple medications, the addition of new pharmacological agents such as SGLT2 inhibitors and GLP1 receptor agonists in the list of essential medicines, and cost-effective population-based interventions to confront the obesity epidemic are all of the essence.

V.M. The Government of India has introduced a very ambitious Ayushman Bharat Scheme under its Niti Ayog programme. Under this scheme, 150,000 Health and Wellness Centres are being set up and the treatment of NCDs like diabetes mellitus will be a major focus of these clinics. About half of these clinics are already operational. The availability of essential drugs is ensured at these centres as well as other primary health centres in the villages. This means that a free supply of the basic antidiabetic oral agents and insulin should become available to the population at large. However, continued education and large-scale awareness programmes are needed to ensure adherence to treatment as diabetes mellitus is a lifelong condition.

K.K. Despite good access to medications in many European countries, there are substantial challenges and wide variations in the uptake of different glucose-lowering therapies across Europe. To improve patient outcomes, there needs to be universal, affordable and timely access to innovative therapies.

M.F. There is no simple solution to improve disparities in diabetes mellitus care, but one potential improvement (albeit incremental) 
resulting from the COVID-19 pandemic is the increased availability of telemedicine. Telemedicine has lowered physical and financial barriers for people to see both general practitioners and specialists. Long-term data are needed to confirm the anecdotal evidence indicating that this approach improves follow-up rates and downstream effects (for example, improved glycaemic control).

A.J.J. Health care is a basic human right, not a privilege. This is underpinned by international human rights laws, including the right to life, the right to health and the right to information, and is based on dignity and the universality of obligation and non-discrimination. Informed by human rights approaches, there must be a greater united multi-pronged approach to improving diabetes mellitus outcomes. Individuals, communities, organizations, governments and NGOs must raise the awareness of all stakeholders, from individual to international level, of the rights and needs of many to diabetes mellitus care. There should be advocacy to Ministries of Health, governments, pharmaceutical and device agencies, and to UN Human Rights Committees and agencies. Universal health coverage is much needed. There is always a moral obligation to treat people with diabetes mellitus; many people, organizations and governments desire to do so, but some will respond more to legal obligations and oversight.

Committed people working together have repeatedly achieved the seemingly impossible. This includes better health for people with HIV and AIDS and the rapid development of effective vaccines against COVID-19. All featured the sharing of resources with people in less advantaged regions. We must unite and accelerate our efforts in Oceania and beyond to prevent diabetes mellitus and to provide equitable access to excellent diabetes mellitus care.

Michael Fralick ${ }^{1 凶}$, Alicia J. Jenkins ${ }^{2,3 凶}$, Kamlesh Khunti ${ }^{4 凶}$, Jean Claude Mbanya ${ }^{5 凶}$, Viswanathan Mohan ${ }^{6}{ }^{6}$ and Maria Inês Schmidt ${ }^{7 凶}$
'Sinai Health System, Department of Medicine, University of Toronto, Toronto, ON, Canada. ${ }^{2}$ NHMRC Clinical Trials Centre, The University of Sydney, Sydney, New South Wales, Australia.

${ }^{3}$ Insulin For Life Global, Sydney, New South Wales, Australia.

${ }^{4}$ Leicester Diabetes Centre, University of Leicester, Leicester, UK.

${ }^{5}$ Faculty of Medicine and Biomedical Sciences, University of Yaoundé I, Yaoundé, Cameroon.

${ }^{6}$ Dr. Mohan's Diabetes Specialities Centre \& Madras Diabetes Research Foundation, Chennai, Tamil Nadu, India.

${ }^{7}$ School of Medicine, Universidade Federal do Rio Grande do Sul, Porto Alegre, RS, Brazil.

凶e-mail:mike.fralick@mail.utoronto.ca; alicia.jenkins@sydney.edu.au; kk22@le.ac.uk; jcmbanya@yahoo.co.uk;drmohans@diabetes.ind.in; maria.schmidt@ufrgs.br

https:/doi org/10.1038/s41574-021-00621-y

Published online 17 January 2022

1. Fralick, M. \& Kesselheim, A. S. The U.S. insulin crisis rationing a lifesaving medication discovered in the 1920s. N. Engl. J. Med. 381, 1793-1795 (2019).

2. Pfiester, E. et al. Costs and underuse of insulin and diabetes supplies: Findings from the 2020 T1 International cross-sectional web-based survey. Diabetes Res. Clin. Pract. 179, 108996 (2021).

3. Herkert, D. et al. Cost-related insulin underuse among patients with diabetes. JAMA Intern. Med. 179, 112-114 (2019).

4. Ewen, M., Joosse, H.-J., Beran, D. \& Laing, R. Insulin prices, availability and affordability in 13 low-incom and middle-income countries. BMJ Glob. Health 4 e001410 (2019).

5. International Diabetes Federation. IDF Diabetes Atlas 10th Edition 2021. Diabetesatlas.org https:/ diabetesatlas.org/atlas/tenth-edition/ (2021).

6. Romer, D. Brazil's efforts to reduce cigarette use illustrate both the potential successes and challenges of this goal. Am. J. Public. Health 111, 549-550 (2021).

7. Malta, D. C. et al. Prevalência de diabetes mellitus determinada pela hemoglobina glicada na população adulta brasileira, Pesquisa Nacional de Saúde. Rev. Bras. Epidemiol. https://doi.org/10.1590/ 1980-549720190006.supl.2 (2019).

8. Dos Reis, R. C. P., Duncan, B. B., Szwarcwald, C. L. Malta, D. C. \& Schmidt, M. I. Control of glucose, blood pressure, and cholesterol among adults with diabetes: the Brazilian national health survey. J. Clin. Med. 10, 3428 (2021)

9. Chow, C. K. et al. Availability and affordability of essential medicines for diabetes across high-income middle-income, and low-income countries: a prospective epidemiological study. Lancet Diabetes Endocrinol. 6, 798-808 (2018).

10. Mardetko, N. et al. Uptake of new antidiabetic medicines in 11 European countries. BMC Endocr. Disord. 21, 127 (2021).

11. Ogle, G. D., Kim, H., Middlehurst, A. C., Silink, M. \& Jenkins, A. J. Financial costs for families of children with type 1 diabetes in lower-income countries. Diabet Med 33, 820-826 (2016).

12. Klatman, E. L., Jenkins, A. J., Ahmedani, M. Y. \& Ogle, G. D. Blood glucose meters and test strips: global market and challenges to access in low-resource settings. Lancet Diabetes Endocrinol. 7, 150-160 (2019).

13. Atun, R. et al. Diabetes in sub-Saharan Africa: from clinical care to health policy. Lancet Diabetes Endocrinol. 5, 622-667 (2017).

14. Kesselheim, A. S., Avorn, J. \& Sarpatwari, A. The high cost of prescription drugs in the United States: origins and prospects for reform. JAMA 316, 858-871 (2016)

15. Fralick, M., Colacci, M., Odutayo, A., Siemieniuk, R. $\&$ Glynn, R. J. Lowering of hemoglobin A1C and risk of cardiovascular outcomes and all-cause mortality, a meta-regression analysis. J. Diabetes Complications 34, 107704 (2020).

16. Unnikrishnan, R., Anjana, R. M. \& Mohan, V. Diabetes mellitus and its complications in India. Nat. Rev. Endocrinol. 12, 357-370 (2016).

17. Khunti, K. et al. Achievement of guideline targets for blood pressure, lipid, and glycaemic control in type 2 diabetes: A meta-analysis. Diabetes Res. Clin. Pract. 137, 137-148 (2018).

18. Gomes, M. B. et al. Treatment of type 2 diabetes mellitus worldwide: Baseline patient characteristics in the global DISCOVER study. Diabetes Res. Clin. Pract. 151, 20-32 (2019).

19. Beran, D., Lazo-Porras, M., Mba, C. M. \& Mbanya, J. C. A global perspective on the issue of access to insulin. Diabetologia 64, 954-962 (2021).

\section{Acknowledgements}

K.K. acknowledges the support of the National Institute for Health Research (NIHR) Applied Research Collaboration East Midlands (ARC EM) and the NIHR Leicester Biomedical Research Centre (BRC).

\section{Competing interests}

M.F. is part of a start-up company called ProofDx that has created a point-of-care testing device using CRISPR for COVID-19. M.F. has received multiple grants from CIHR and the Canadian Military for clinical trials to identify treatments for COVID-19. A.J.J. has received peer-reviewed diabetes-related grants from the following bodies: NHMRC Australia, JDRF Australia, JDRF International, Abbott Europe, Sanofi-Aventis, and Sydney Medical School and Foundation. A.J.J. is an honorary board member of Insulin for Life, a founder/co-chair of the Diabetes Advocacy Group, including members of Insulin for Life, the Life for a Child programme and Children Living as Neighbours, a member of the International Diabetes Federation (IDF) Western Pacific Executive Council, Australia's representative to the IDF and elected to the IDF Western Pacific Executive Council. K.K. has acted as consultant, advisory board member and speaker for Abbott, Amgen, AstraZeneca, Bayer, NAPP, Lilly, Merck Sharp and Dohme, Novartis, Novo Nordisk, Roche, Berlin-Chemie AG/Menarini Group, Sanofi-Aventis, Servier and Boehringer Ingelheim, and EACME grants from Boehringer Ingelheim, AstraZeneca, Novartis, Novo Nordisk, Sanofi-Aventis, Lilly, and Merck Sharp \& Dohme. J.C.M. and the institutions to which he is associated have received funding from the following pharmaceutical companies for educational, advisory and research activities: AstraZeneca, GSK, Novartis, Novo Nordisk, Sanofi and Servier. V.M. has acted as consultant and speaker and received research or educational grants from Novo Nordisk, MSD, Eli Lilly, AstraZeneca, Novartis, Boehringer Ingelheim, Lifescan J\&J, Sanofi-Aventis, Roche Diagnostics, Abbott and from several Indian pharmaceutical companies including USV, Dr. Reddy's Laboratories and Sun Pharma. M.I.S. declares no competing interests.

\section{Publisher's note}

Springer Nature remains neutral with regard to jurisdictional claims in published maps and institutional affiliations.

C) Springer Nature Limited 2022 